\title{
Bar pattern speeds in CALIFA galaxies
}

\section{The case of weakly barred galaxies}

\author{
Virginia Cuomo ${ }^{1}$, J. Alfonso Lopez Aguerri ${ }^{2,3}$, Enrico Maria Corsini ${ }^{1,4}$, Victor P. Debattista ${ }^{5}$, \\ Jairo Méndez-Abreu ${ }^{2,3}$, and Alessandro Pizzella ${ }^{1,4}$ \\ 1 Dipartimento di Fisica e Astronomia "G. Galilei”, Università di Padova, Vicolo dell'Osservatorio 3, 35122 Padova, Italy \\ e-mail: virginia.cuomo@phd.unipd.it \\ 2 Departamento de Astrofísica, Universidad de La Laguna, Avenida Astrofísico Francisco Sánchez s/n, 38206 La Laguna, Tenerife, \\ Spain \\ 3 Instituto de Astrofísica de Canarias, Calle Vía Láctea s/n, 38205 La Laguna, Tenerife, Spain \\ ${ }^{4}$ INAF - Osservatorio Astronomico di Padova, vicolo dell'Osservatorio 2, 35122 Padova, Italy \\ 5 Jeremiah Horrocks Institute, University of Central Lancashire, PR1 2HE Preston, UK
}

Received 31 July 2019 / Accepted 2 September 2019

\begin{abstract}
Context. About $35 \%$ of the nearby disc galaxies host a weak bar for which different formation scenarios, including the weakening of a strong bar and tidal interaction with a companion, have been suggested. Measuring the bar pattern speeds of a sample of weakly barred galaxies is a key step in constraining their formation process, but such a systematic investigation is still missing.

Aims. We investigated the formation process of weak bars by measuring their properties in a sample of 29 nearby weakly barred galaxies, spanning a wide range of morphological types and luminosities. The sample galaxies were selected to have an intermediate inclination, a bar at an intermediate angle between the disc minor and major axes, and an undisturbed morphology and kinematics to allow the direct measurement of the bar pattern speed. Combining our analysis with previous studies, we compared the properties of weak and strong bars.

Methods. We measured the bar radius and strength from the $r$ band images available in the Sloan Digital Sky Survey and bar pattern speed and corotation radius from the stellar kinematics obtained by the Calar Alto Legacy Integral Field Area Survey. We derived the bar rotation rate as the ratio between the corotation and bar radii.

Results. Thirteen out of 29 galaxies (45\%), which were morphologically classified as weakly barred from a visual inspection, do not actually host a bar component or their central elongated component is not in rigid rotation. We successfully derived the bar pattern speed in 16 objects. Two of them host an ultrafast bar. Using the bar strength to differentiate between weak and strong bars, we found that the weakly barred galaxies host shorter bars with smaller corotation radii than their strongly barred counterparts. Weak and strong bars have similar bar pattern speeds and rotation rates, which are all consistent with being fast. We did not observe any difference between the bulge prominence in weakly and strongly barred galaxies, whereas nearly all the weak bars reside in the disc inner parts, contrary to strong bars.

Conclusions. We ruled out that the bar weakening is only related to the bulge prominence and that the formation of weak bars is triggered by the tidal interaction with a companion. Our observational results suggest that weak bars may be evolved systems exchanging less angular momentum with other galactic components than strong bars.
\end{abstract}

Key words. galaxies: evolution - galaxies: formation - galaxies: general - galaxies: kinematics and dynamics - galaxies: structure

\section{Introduction}

Bars are so common in the centre of disc galaxies that in the most widely adopted morphological classifications (Hubble 1936; de Vaucouleurs 1959; van den Bergh 1976), barred galaxies represent one of the main families of both lenticular and spiral galaxies. Since bars show a wide variety of properties in terms of size, luminosity, and shape, disc galaxies are divided into unbarred, weakly barred, and strongly barred galaxies.

Now it is known that bars are hosted in $\sim 50 \%$ of galactic discs in the local universe if observed in the optical bands, and this fraction rises to $\sim 70 \%$ in the near-infrared (Aguerri et al. 2009; Buta et al. 2015) and about half of them are classified as weak bars (Buta et al. 2007). The bar fraction in nearby galaxies depends on the morphological type (Marinova \& Jogee 2007; Aguerri et al. 2009; Li et al. 2017) and is a strong function of the galaxy luminosity (or equivalently stellar mass) since it peaks for giant galaxies and decreases in both the low- and high-mass regimes (Méndez-Abreu et al. 2010; Nair \& Abraham 2010; Sánchez-Janssen et al. 2010). The bar fraction distribution as a function of galaxy luminosity varies significantly from cluster to field environments (Barway et al. 2011; Méndez-Abreu et al. 2012; Lin et al. 2014). A very weak trend is found between bar fraction and colour, slowly declining to redder colours (Barazza et al. 2008), although there are conflicting results (Masters et al. 2011; Erwin 2018).

The radius $R_{\text {bar }}$, strength $S_{\text {bar }}$, and pattern speed $\Omega_{\text {bar }}$ are the main properties of a bar. The bar radius defines the extension of the stellar orbits supporting the bar, which mainly belong to the highly elongated $x_{1}$ family (Contopoulos \& Papayannopoulos 1980; Manos \& Athanassoula 2011), even if a fraction of stochastic orbits is also theoretically predicted (Martinet \& Udry 1990; 
Patsis \& Katsanikas 2014). The bar strength measures the nonaxisymmetric forces produced by the bar potential (Laurikainen $\&$ Salo 2002). The bar pattern speed is the angular frequency with which the bar rotates around the galactic centre (Athanassoula 2003; Combes 2011). This parameter is related to both the light and mass distribution of the host galaxy since it mainly depends on the redistribution of angular momentum between the galactic components (Debattista \& Sellwood 2000; Athanassoula 2003). The bar pattern speed is usually parametrised by the bar rotation rate $\mathcal{R}=R_{\mathrm{cr}} / R_{\mathrm{bar}}$, where $R_{\mathrm{cr}}$ is the corotation radius, where the gravitational attraction balances the centrifugal acceleration in the rest-frame of the bar. Bars having $1.0 \leq \mathcal{R} \leq 1.4$ end close to corotation and they are called "fast". In contrast, bars with $\mathcal{R}>1.4$ are shorter than corotation and they are termed "slow" (Debattista \& Sellwood 1998, 2000; Athanassoula \& Misiriotis 2002). Bars with $\mathcal{R}<1.0$ corresponds to an unphysical regime for the $x_{1}$ orbits (Contopoulos 1981; Athanassoula 1992; Vasiliev \& Athanassoula 2015) and are called "ultrafast" (Buta \& Zhang 2009; Aguerri et al. 2015; hereafter Paper I).

The formation of a bar in an isolated galaxy is generally attributed to internal processes and typically includes three main phases: the initial growth, subsequent buckling, and final secular evolution (e.g. Hohl 1971; Noguchi 1987; Sellwood 1981; Toomre 1981; Raha et al. 1991; Debattista et al. 2006; Athanassoula et al. 2013; Martinez-Valpuesta et al. 2017). The bar growth takes $\sim 2 \mathrm{Gyr}$, at the end of which a clear nonaxysimmetric stellar structure stands out in the disc. During the buckling phase, which lasts $\sim 1 \mathrm{Gyr}$, the bar weakens. The following secular evolution takes place over several Gyr, and the bar slowly increases its length and strength. The bar pattern speed decreases at a rate depending on the amount of angular momentum exchanged between the disc and other galactic components and on the dynamical friction exerted on the bar by the dark matter (DM) halo. Measuring the bar rotation rate allows us to investigate the bar evolution and at the same time to constrain the DM distribution (Debattista \& Sellwood 2000; Athanassoula 2003). Other external events, such as interactions with companions and satellites (Athanassoula et al. 2013; Martinez-Valpuesta et al. 2016; Łokas 2018), and internal processes such the gas fraction, the shape of DM halo, and the presence of a central mass concentration (Athanassoula 2003; Debattista et al. 2006; Athanassoula et al. 2013) further influence the formation and evolution of a bar. More specifically, Bournaud \& Combes (2002) and Bournaud et al. (2005) found that a significant gas accretion in the presence of a massive bulge produces different episodes of bar destruction and rebuilding. At each step, the newly formed bar is shorter and weaker, while its pattern speed is faster than the previous one.

A first pioneering effort to explain the formation of weak bars was made by Kormendy (1979), who concluded that lenses are the end result of the evolution of bars into nearly axisymmetric structures. Since the fraction of barred galaxies hosting a lens is very high, the mechanism should be secular and possibly involve the interaction with the bulge. Moreover, the majority of lenses are located in early-type galaxies with large central concentrations. This is in conflict with the findings of Laurikainen et al. (2013), who found that the radius of fully developed lenses is on average $\sim 1.3$ times larger than that of bars. Recently, Kruk et al. (2018) have provided some evidence supporting the idea that bars dissolve into lenses. They found that unbarred discs are bluer than their barred counterparts, while unbarred galaxies with a lens are similar to strongly barred galaxies. Weakly barred galaxies are very similar to unbarred galaxies since their discs are bluer and their bars are shorter than those in strongly barred galaxies (Abraham et al. 1999). Weak bars can also represent the end result of weak interactions. Martinez-Valpuesta et al. (2017) investigated, through numerical simulations, the formation of bars triggered or affected by fast interactions. These bars formed by interactions are slow throughout their lifetime. Low values of the bar pattern speed (corresponding to $\mathcal{R} \sim 2$ ) have been also found by Łokas (2018) in the late evolutionary stages of tidally induced bars.

Therefore measuring the bar pattern speed of a sample of weakly barred galaxies could constrain their formation process. This is a challenging task; in the past a variety of indirect methods based on modelling was used to measure the pattern speed and corresponding rotation rates of strong bars. The only modelindependent method proposed to date is the one by Tremaine \& Weinberg (1984; hereafter TW). In the last two decades, the applications of the TW method using long-slit spectroscopic data of stellar kinematics allowed the study of $\sim 20$ galaxies (see Corsini 2011, for a review). More recently, integral-field spectroscopy has been shown to remarkably improve the efficiency and precision of the TW measurements. In Paper I we measured the bar pattern speed of 15 galaxies on the stellar velocity maps provided by Calar Alto Legacy Integral Field Area survey (CALIFA; Sánchez et al. 2012), and Guo et al. (2019) obtained the bar pattern speed for another 51 galaxies using the integralfield spectroscopic data from the Massive Nearby Galaxies survey (MaNGA; Bundy et al. 2015). However, neither Paper I nor Guo et al. (2019) included weakly barred galaxies in their samples. To date, the bar pattern speed has been measured with the TW method in only one weakly barred galaxy, ESO 139-G0009, which turned out to host a fast bar (Aguerri et al. 2003). The bar pattern speed has been indirectly measured from the velocity field of the ionised or molecular gas in a number of weakly barred galaxies suggesting that slow bars are hosted especially by late-type spirals in spite of large uncertainties on $\mathcal{R}$ (Hirota et al. 2009; Font et al. 2017; Salak et al. 2019).

In this paper our aim is to investigate the formation of weak bars by measuring the bar radius, strength, and pattern speed with the TW method in a sample of weakly barred galaxies for which integral-field spectroscopic data are available from the CALIFA survey. The paper is structured as follows. We present the galaxy sample in Sect. 2. We measure the bar properties of the sample galaxies in Sect. 3. We present our results in Sect. 4. We discuss our conclusions in Sect. 5. We adopt as cosmological parameters $\Omega_{\mathrm{m}}=0.286, \Omega_{\Lambda}=0.714$, and $H_{0}=69.3 \mathrm{~km} \mathrm{~s}^{-1} \mathrm{Mpc}^{-1}$ (Hinshaw et al. 2013).

\section{Sample selection}

In this work we analyse the bar properties for a large sample of galaxies with a TW-measured $\Omega_{\text {bar }}$ and spanning a wide range of bar strengths. Several strongly barred galaxies have been already measured in the literature, but there is a lack of weak bars. To fill this gap we took galaxies from the CALIFA survey, whose aim was to measure the properties of a statistically significant sample of nearby galaxies with integral field spectroscopy (Sánchez et al. 2012). The CALIFA Data Release 3 (DR3; Sánchez et al. 2016) includes 700 galaxies from the SDSS Data Release 7 (SDSS-DR7; Abazajian et al. 2009) selected to have a major-axis diameter $45<D_{25}<80$ arcsec in the $r$ band and a redshift $0.005<z<0.03$. They were observed with the Potsdam Multi-Aperture Spectrograph (PMAS; Roth et al. 2005) mounted at the $3.5 \mathrm{~m}$ telescope of the Calar Alto Observatory (Husemann et al. 2013; Walcher et al. 2014). We considered the 265 CALIFA galaxies that were morphologically classified as 
doubtful barred galaxies (Walcher et al. 2014). The visual identification of bars is not always obvious and it is even more difficult in the case of weakly barred galaxies. The morphological classification performed by the CALIFA collaboration does not always match the de Vaucouleurs et al. (1991; hereafter RC3) classification (Table 1).

From the CALIFA SAB galaxies we selected those for which the stellar kinematic maps were measured by Falcón-Barroso et al. (2017). These selection criteria allowed us to discard a priori the objects with a disturbed kinematics. We remained with 58 galaxies visually classified as SAB, for which we analysed the stellar kinematics obtained with a spectral resolution of $R=1650$ (corresponding to $\sigma_{\text {inst }} \sim 70 \mathrm{~km} \mathrm{~s}^{-1}$ at $4500 \AA$ ) and a spatial resolution of 1 arcsec.

The TW method allows us to measure $\Omega_{\mathrm{bar}}$ from

$\langle X\rangle \Omega_{\mathrm{bar}} \sin i=\langle V\rangle$,

where $i$ is the disc inclination, and

$\langle X\rangle=\frac{\int X \Sigma \mathrm{d} \Sigma}{\int \Sigma \mathrm{d} \Sigma} ;\langle V\rangle=\frac{\int V_{\mathrm{LOS}} \Sigma \mathrm{d} \Sigma}{\int \Sigma \mathrm{d} \Sigma}$

are the photometric and kinematic integrals, defined as the luminosity-weighted average of position $X$ and line-of-sight (LOS) velocity $V_{\text {LOS }}$, respectively, while $\Sigma$ represents the surface brightness of the galaxy. They have to be measured along directions parallel to the disc major axis. Then, fitting the values of $\langle X\rangle$ and $\langle V\rangle$ obtained for different offset positions crossing the bar with a straight line then gives $\Omega_{\text {bar }} \sin i$, so at least two pseudoslits have to be defined. The use of integral-field spectroscopic data allows the measurement of $\langle X\rangle$ and $\langle V\rangle$ in several parallel pseudoslits by collapsing their corresponding spectra along the spectral and spatial directions, respectively. To this end we followed the prescriptions given in Paper I.

In order to apply any further analysis on our sample, we needed to have the structural parameters of the galaxies. We selected the 37 sample galaxies whose SDSS $r$ band images were analysed by Méndez-Abreu et al. (2017). They performed the isophotal analysis and photometric decomposition of the surface brightness distribution using the IRAF task ELLIPSE (Jedrzejewski 1987) and GASP2D (Méndez-Abreu et al. 2008, 2014), respectively.

Moreover, to successfully apply the TW method, the galaxies should have an intermediate inclination and their bars should be elongated at an intermediate position angle (PA) between the disc major and minor axes. Low-inclination galaxies are characterised by small stellar velocities, large velocity errors, and a large uncertainty on the disc PA, while in highly inclined galaxies it is difficult to identify the bar and to locate the pseudoslits. A bar aligned with the disc major axis gives $\langle X\rangle=0$ arcsec, while a bar aligned with the disc minor axis is characterised by $\langle V\rangle=V_{\text {sys. }}$. To address these issues we rejected all the galaxies with a $\triangle \mathrm{PA}<10^{\circ}$ between the bar major axis and disc major or minor axis and kept objects with a disc inclination $25^{\circ}<i<75^{\circ}$, as done in Paper I. When the photometric decomposition did not include a bar component, we recovered the bar PA from the analysis of the ellipticity $\epsilon$ and PA radial profiles, as discussed in Sect. 3.1. In the end, our sample of bona fide SAB galaxies totals 29 objects, whose main properties are listed in Table 1. The distributions of their morphological types, redshifts, and absolute SDSS $r$ band magnitudes are plotted in Fig. 1. Since the distribution in morphologies, redshift, and absolute magnitudes reflect the properties of the mother sample in CALIFA (Walcher et al.
2014), we can conclude there is no bias in our final selected sample with respect to the initial one.

In addition, the TW method works for a stellar tracer satisfying the continuity equation and because of this it was initially applied to early-type disc galaxies, which do not show strong evidence of spiral arms or heavily patchy dust distribution. Spiral arms may lead to an incorrect determination of the disc PA, and their light contribution may affect the photometric integrals of the bar. The presence of dust and/or star formation may cause a non-coincidence between the surface brightness and mass distribution of the galaxy, which results in a mismatch between photometric and kinematic measurements. These effects may be mitigated by computing the mass-weighted kinematic and photometric integrals (Gerssen \& Debattista 2007). However, Paper I compared the values of $\Omega_{\text {bar }}$ derived for a number of spirals from both light- and mass-weighted TW integrals and found consistent results even in late-type galaxies, which are the most affected by this problem. This means that the TW method can be applied to barred spirals after checking the convergence of the TW integrals, which allows us to control and limit contamination from other spurious features, such as foreground stars or bad pixels.

\section{Properties of the weak bars}

\subsection{Disc inclination and position angle}

The TW method is very sensitive to the misalignment between the orientation of pseudoslits and $\langle V\rangle$ and disc major axis. In addition, the calculation of $\Omega_{\text {bar }}$ also requires knowing the disc inclination (Debattista 2003).

In order to accurately constrain the disc orientation, we decided to consider both the photometric decomposition and isophotal analysis of the SDSS $r$ band images provided by Méndez-Abreu et al. (2017). For each object, we derived the disc inclination $i$ and PA from the ellipticity $\epsilon$ and major-axis PA of the ellipses fitting the outermost galaxy isophotes measured by Méndez-Abreu et al. (2017). We defined the extension of the disc radial range by fitting the PA measurements with a straight line and selecting all the radii where the linear slope was consistent with zero within the associated root mean square error. We adopted the mean PA and mean $\epsilon$ and corresponding root mean square errors as the disc geometric parameters and their errors. Finally, we derived $i=\arccos (1-\epsilon)$ by assuming an infinitesimally thin disc.

The resulting PA values are not always consistent within the $3 \sigma$ errors with those from the photometric decomposition by Méndez-Abreu et al. (2017). Although the mean difference of $\triangle \mathrm{PA}$ is lower than 1.5 in $80 \%$ of the sample, for a few galaxies the difference is as large as $\triangle \mathrm{PA} \sim 7^{\circ}$. It should be noted that the bar component was not always included in the photometric decomposition due to the weakly barred nature of these galaxies and this affects the resulting best-fitting parameters of the disc (see Méndez-Abreu et al. 2014; de Lorenzo-Cáceres et al. 2019, for a discussion). For each galaxy, we defined a range for the disc PA which covers the values from the photometric decomposition and isophotal analysis and their errors to be used for the application of the TW method. The $i$ values from the isophotal analysis are consistent with those from the photometric decomposition listed in Table 1, which we adopt here.

\subsection{Bar detection}

The visual identification of weakly barred galaxies is difficult (e.g. Nair \& Abraham 2010; Lee et al. 2019) and most of our 


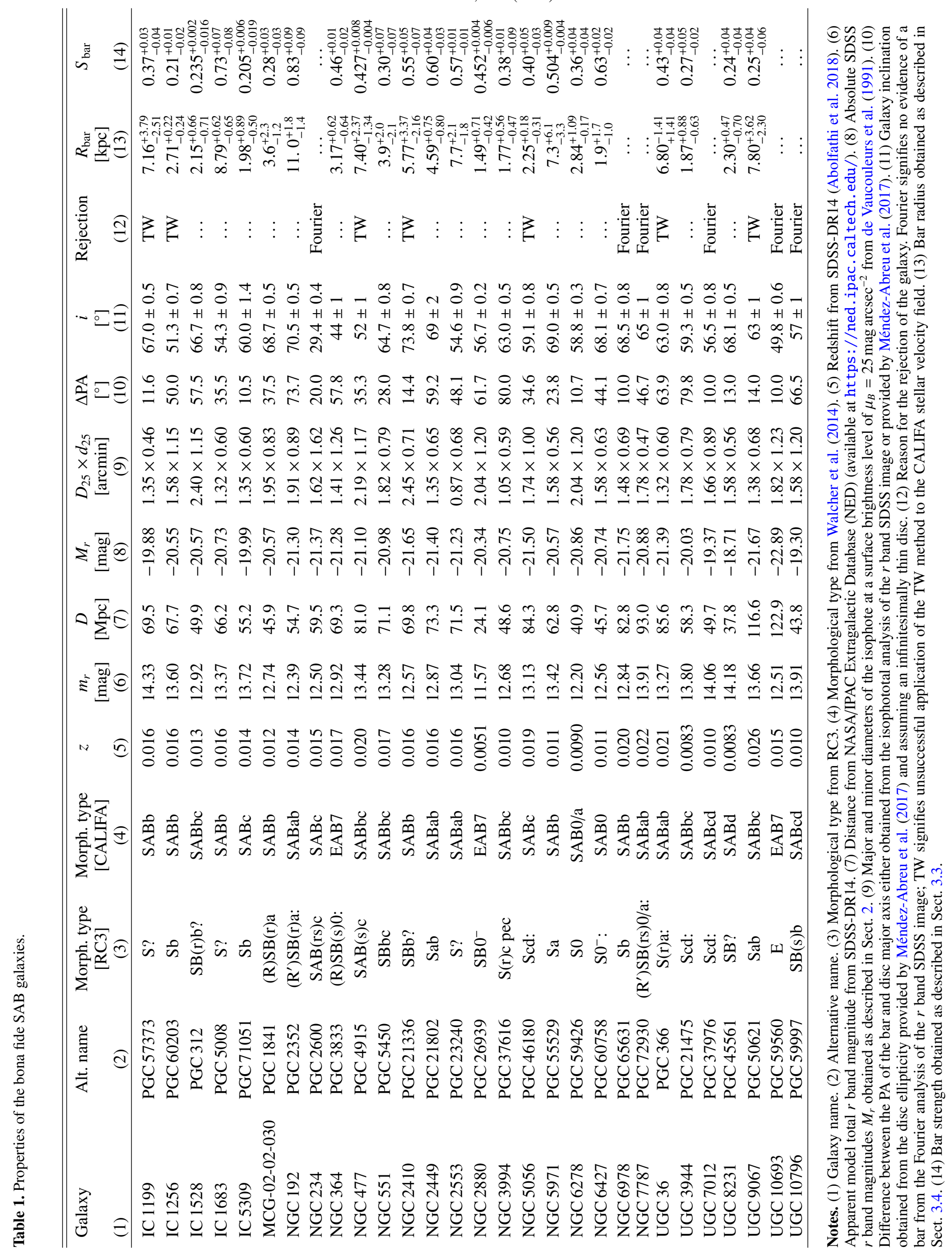




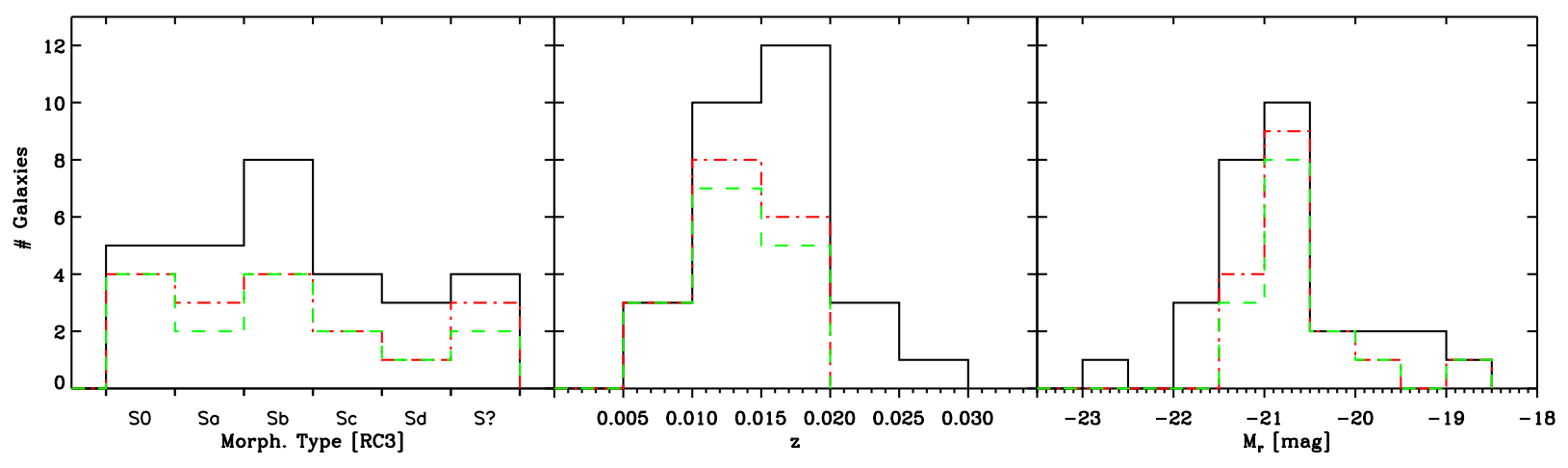

Fig. 1. Distribution of the morphological type (left panel), redshift (central panel), and absolute $r$ band magnitude (right panel) of our sample of 29 bona fide SAB galaxies (black solid line), the sample of 16 SAB galaxies successfully analysed with the TW method (red dot-dashed line), and the $14 \mathrm{SAB}$ galaxies hosting a non-ultrafast bar (green dashed line).

bona fide/SAB galaxies were actually listed in RC3 either as unbarred galaxies or their classification was uncertain (Table 1). For this reason, the accurate analysis of the galaxy surface brightness distribution is a mandatory step to identify the presence of a genuine bar component.

The Fourier analysis of the light distribution has often been used to detect and characterise the different galactic components, especially bars, which correspond to bisymmetric departures from axisymmetry (Ohta et al. 1990; Athanassoula 2003; Garcia-Gómez et al. 2017). Following Aguerri et al. (2000), we decomposed the deprojected azimuthal surface brightness profile $I(r, \phi)$ of each sample galaxy, where $(r, \phi)$ are the polar coordinates in the galaxy disc. We recovered the deprojected $r$ band image of the galaxy by stretching the original SDSS image along the disc minor axis by a factor equal to $1 / \cos i$, with the flux conserved. We adopted the geometric parameters of the disc derived by Méndez-Abreu et al. (2017).

We know that the bar region is characterised by large values of the even Fourier components, and in particular of the $m=2$ Fourier component. The odd Fourier components are generally smaller than the even ones because they are associated with the presence of asymmetric components. The maximum amplitude of the $m=2$ Fourier component is correlated with the bar strength and the bars typically have $\left(I_{2} / I_{0}\right)_{\max }>0.2$ (Aguerri et al. 2003). We expect that the phase angle $\phi_{2}$ of the $m=2$ Fourier component is constant within the bar region. We found that the Fourier components of six sample galaxies do not meet these criteria. Therefore, we concluded that these galaxies are more likely to be unbarred systems and we excluded them from the analysis (Table 1).

\subsection{Bar strength}

As in Paper I, we measured $S_{\text {bar }}=\left(I_{2} / I_{0}\right)_{\max }$ for all the weak bar sample galaxies as the maximum of the intensity ratio between the $m=2$ and $m=0$ Fourier components (Athanassoula \& Misiriotis 2002). The uncertainties associated with the measurement of the strength are obtained by performing a Fourier analysis using the two portions of the deprojected azimuthal surface brightness $I(r, \phi)$ with $0^{\circ}<\phi<180^{\circ}$ and $180^{\circ}<\phi<360^{\circ}$. The difference between these two measurements with respect to the reference value obtained from the full surface brightness distribution provided the errors on the bar strength, typically smaller than $10 \%$. The resulting values $S_{\text {bar }}$ and corresponding errors are reported in Table 1. We define in Sect. 4.2 a quantitative criterion based on $S_{\text {bar }}$ to distinguish between weak and strong bars.

\subsection{Bar radius}

For each of the 23 galaxies confirmed as hosting a weak bar, we measured $R_{\mathrm{bar}}$ from the analysis of the SDSS $r$ band image using three independent methods, as done in Paper I. We considered the bar-to-interbar intensity ratio obtained from the Fourier analysis, the location of the maximum in the $\epsilon$ radial profile, and the behaviour of the PA radial profile. The radial profiles of $\epsilon$ and PA are derived by fitting ellipses to the isophotes of the $r$ band image using ELLIPSE and considering a variable value for the centre of the galaxy (Méndez-Abreu et al. 2017).

First, we measured $R_{\text {bar }}$ from the Fourier analysis by tracing the radial profile of the intensity contrast between the bar and interbar regions. Following Aguerri et al. (2000), we defined the bar intensity as $I_{\mathrm{bar}}=I_{0}+I_{2}+I_{4}+I_{6}$ and the interbar intensity as $I_{\text {ibar }}=I_{0}-I_{2}+I_{4}-I_{6}$. The bar region corresponds to the radial range where the bar-to-interbar intensity ratio is $I_{\text {bar }} / I_{\text {ibar }}>0.5\left[\max \left(I_{\text {bar }} / I_{\mathrm{ibar}}\right)-\min \left(I_{\mathrm{bar}} / I_{\mathrm{ibar}}\right)\right]+\min \left(I_{\mathrm{bar}} / I_{\mathrm{ibar}}\right)$. We adopted the FWHM of the radial profile of $I_{\mathrm{bar}} / I_{\mathrm{ibar}}$ as the bar radius.

Then, we measured $R_{\text {bar }}$ from the $\epsilon$ radial profile of the isophotal ellipses since it traces the shape and size of the stellar orbits supporting the bar. The galaxy isophotes usually appear almost circular near the centre, while their $\epsilon$ increases up to a local maximum in the bar region and decreases outwards to a local minimum in the disc region. Following Wozniak \& Pierce (1991), we adopted the position of the maximum $\epsilon$ as second estimate of $R_{\text {bar }}$.

Finally, we obtained a third estimate of $R_{\mathrm{bar}}$ from the analysis of the radial profile of the PA with the isophotal ellipses. The galaxy isophotes show a constant PA in the bar and disc regions. Usually, the two values are different because they are related to the orientation of the bar and the line of nodes, respectively. As in Paper I, we adopted as $R_{\mathrm{bar}}$ the position where the PA changes by $\Delta \mathrm{PA}=5^{\circ}$ from the PA of the ellipse with the maximum $\epsilon$ value.

We adopted for each galaxy the mean value from the three measurements and the largest deviation from the highest and lowest estimates from the mean as $R_{\mathrm{bar}}$ and corresponding upper and lower errors, respectively. This corresponds to a typical error around $30 \%$. The resulting values of $R_{\mathrm{bar}}$ and error are listed in Table 1. 

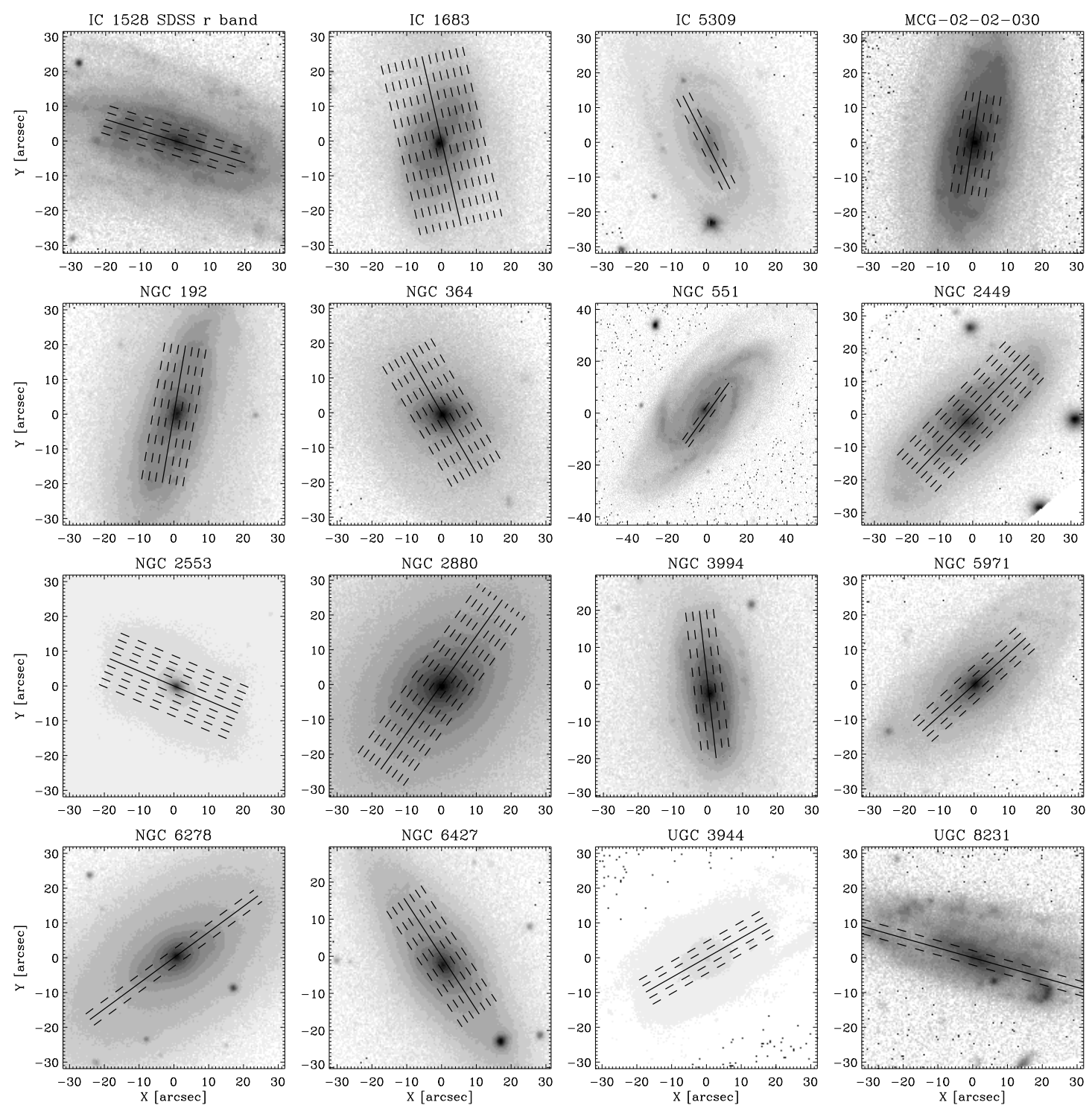

Fig. 2. SDSS $r$ band images of the sample of 16 SAB galaxies successfully analysed with the TW method. The FOV is oriented with north up and east left. For each galaxy, the position and length of the pseudoslits that are parallel to the disc major axis and cross the galaxy centre (solid line) or are offset with respect to it (dashed lines) are shown.

\subsection{Bar pattern speed}

We applied the TW method, as outlined in Eq. (1). For each galaxy the pseudoslits were defined a posteriori from the CALIFA reconstructed image. We traced from 3 to 13 pseudoslits that were 1 arcsec in width crossing the bar and oriented with the disc PA. A minimum separation of 1 arcsec between adjacent pseudoslits was fixed to deal with independent data and minimise the impact of spatial correlations on the TW integrals. We adopted a half length of the pseudoslits in the range of (1-4) $h$, where $h$ is the exponential scale length of the disc from the photometric decompositions of Méndez-Abreu et al. (2017). In all cases we checked that the pseudoslits extended out to the axisymmetric region of the disc defined from the photometric decomposition (Fig. 2).

We measured $\langle X\rangle$ in the CALIFA reconstructed image obtained by summing the CALIFA datacube along the spectral direction in the wavelength range between 4500 and $4650 \AA$ and excluding intervals severely affected by bad pixels. The spectral range was selected because it does not cover prominent emission lines. Errors on $\langle X\rangle$ in each slit were defined

as the standard deviation of the $\langle X\rangle$ measured varying the slit length within the range of the constant behaviour of each integrals. The errors typically range between 0.07 and 0.15 arcsec, similar to what was found in Paper I. The convergence of the photometric integrals was checked by measuring them as a function of the coordinates of the galaxy centre and the pseudoslit length.

We measured $\langle V\rangle$ by collapsing each pseudoslit along the spatial direction and measuring the LOS velocity of the resulting spectrum in the wavelength range between $3400 \AA$ and $4750 \AA$ with the Penalized Pixel Fitting (PPXF; Cappellari \& Emsellem 2004) and Gas and Absorption Line Fitting (GANDALF, Sarzi et al. 2006) IDL $^{1}$ algorithms. Since the kinematic integrals are not affected by corrupted pixels at the ends of the spectral intervals, the entire CALIFA spectral range was adopted to recover them. This approach is equivalent to using an explicit luminosity weight because the spaxels with higher signal give higher contribution in the collapsed spectrum and consequently

1 Interactive Data Language is distributed by Harris Geospatial Solutions. 
V. Cuomo et al.: Bar pattern speeds in CALIFA galaxies. II.

Table 2. Properties of the bar and disc of the SAB galaxies successfully analysed with the TW method.

\begin{tabular}{lccccc}
\hline \hline Galaxy & $\begin{array}{c}\mathrm{PA}_{\mathrm{TW}} \\
{\left[{ }^{\circ}\right]}\end{array}$ & $\begin{array}{c}\Omega_{\mathrm{bar}} \\
{\left[\mathrm{km} \mathrm{s}^{-1} \mathrm{kpc}^{-1}\right]}\end{array}$ & $\begin{array}{c}V_{\text {circ }} \\
{\left[\mathrm{km} \mathrm{s}^{-1}\right]}\end{array}$ & $\begin{array}{c}R_{\mathrm{cr}} \\
{[\mathrm{kpc}]}\end{array}$ & $\mathcal{R}$ \\
$(1)$ & $(2)$ & $(3)$ & $(4)$ & $(5)$ & $(6)$ \\
\hline IC 1528 & 72.7 & $87 \pm 20$ & $142 \pm 14$ & $1.63 \pm 0.51$ & $0.76_{-0.22}^{+0.14}$ \\
IC 1683 & 13.0 & $30.3 \pm 5.1$ & $191 \pm 45$ & $6.3 \pm 2.7$ & $0.71_{-0.21}^{+0.21}$ \\
IC 5309 & 26.7 & $91 \pm 26$ & $114 \pm 25$ & $1.25 \pm 1.01$ & $0.63_{-0.45}^{+0.36}$ \\
MCG-02-02-030 & 171.1 & $43.4 \pm 6.5$ & $210 \pm 55$ & $4.83 \pm 2.16$ & $1.32_{-0.53}^{+0.36}$ \\
NGC 192 & 170.4 & $20.9 \pm 2.1$ & $248.3 \pm 6.6$ & $11.9 \pm 1.9$ & $1.08_{-0.13}^{+0.10}$ \\
NGC 364 & 29.9 & $120 \pm 31$ & $317 \pm 30$ & $2.63 \pm 1.13$ & $0.83_{-0.26}^{+0.22}$ \\
NGC 551 & 137.0 & $45 \pm 11$ & $202 \pm 43$ & $4.52 \pm 2.39$ & $1.17_{-0.71}^{+0.39}$ \\
NGC 2449 & 136.4 & $40.7 \pm 5.5$ & $236.9 \pm 2.6$ & $5.84 \pm 0.99$ & $1.27_{-0.14}^{+0.11}$ \\
NGC 2553 & 67.0 & $68.1 \pm 9.8$ & $269 \pm 34$ & $3.95 \pm 0.91$ & $0.515_{-0.110}^{+0.077}$ \\
NGC 2880 & 144.6 & $190 \pm 28$ & $209 \pm 15$ & $1.09 \pm 0.36$ & $0.74_{0.19}^{+0.15}$ \\
NGC 3994 & 6.9 & $119 \pm 27$ & $226.4 \pm 5.5$ & $1.90 \pm 0.67$ & $1.06_{-0.31}^{+0.22}$ \\
NGC 5971 & 132.0 & $55 \pm 15$ & $226 \pm 16$ & $4.07 \pm 1.96$ & $0.56_{-0.32}^{+0.15}$ \\
NGC 6278 & 126.4 & $92 \pm 28$ & $279 \pm 13$ & $3.05 \pm 1.06$ & $1.07_{-0.25}^{+0.26}$ \\
NGC 6427 & 34.7 & $46 \pm 10$ & $245 \pm 21$ & $5.3 \pm 3.6$ & $2.8_{-1.8}^{+1.0}$ \\
UGC 3944 & 119.6 & $62 \pm 22$ & $148 \pm 30$ & $2.39 \pm 11.6$ & $1.28_{-5.7}^{+3.8}$ \\
UGC 8231 & 74.2 & $58 \pm 31$ & $136 \pm 27$ & $2.3 \pm 5.3$ & $1.01_{-2.0}^{+1.6}$ \\
\hline
\end{tabular}

Notes. (1) Galaxy name. (2) Adopted value of the position angle of the pseudoslits for the TW analysis. (3) Bar pattern speed. (4) Disc circular velocity. (5) Bar corotation radius. (6) Bar rotation rate.

in the $\langle V\rangle$ determination of each pseudoslit. We convolved a linear combination of $\sim 330$ stellar spectra available in the IndoUS library (Valdes et al. 2004) with a line-of-sight velocity distribution (LOSVD) modelled as a truncated Gauss-Hermite series (Gerhard 1993; van der Marel \& Franx 1993) via a $\chi^{2}$ minimisation. The stellar spectra were selected to fully cover the parameter space of the effective temperature $T_{\text {eff }}$, surface gravity $g$, and metallicity $[\mathrm{Fe} / \mathrm{H}]$, broadened to match the CALIFA instrumental resolution. After rebinning the stellar spectra to a logarithmic scale along the dispersion direction, we redshifted them to rest frame and cropped their wavelength range to match the redshifted frame of the galaxy spectra. Moreover, a low-order multiplicative Legendre polynomial was added to correct for the different shape of the continuum of the spectra of the galaxy and optimal template due to reddening and large-scale residuals of flat-fielding and sky subtraction. The statistical errors on the stellar kinematic parameters were assumed to be the formal errors of the PPXF best fit after rescaling the minimum $\chi^{2}$ to achieve $\chi_{\min }^{2}=N_{\text {d.o.f. }}=N_{\text {d }}-N_{\text {fp }}$, with $N_{\text {d.o.ff }}, N_{\text {d }}$, and $N_{\text {fp }}$ the number of the degrees of freedom, data points, and fitting parameters, respectively (Press et al. 1992). Errors on $\langle V\rangle$ range between 1 and $15 \mathrm{~km} \mathrm{~s}^{-1}$. The convergence of the kinematic integrals was checked by measuring them as a function of the coordinates of the galaxy centre and pseudoslit length.

The value of $\Omega_{\mathrm{bar}} \sin i$ was obtained by fitting a straight line to the values of $\langle X\rangle$ and $\langle V\rangle$ using the IDL task FITEXY (Fig. 3). We calculated the value of $\Omega_{\mathrm{bar}}$ for both the PAs obtained with the photometric decomposition and isophotal analysis of the galaxy surface brightness distribution and adopting the corresponding values for $i$. For each PA, the TW integrals were measured as outlined above. For each galaxy, we obtained two estimates of $\Omega_{\mathrm{bar}}$, which are in most of the cases compatible within $1 \sigma$ of each other. Nevertheless, we have three cases where the $\Omega_{\text {bar }}$ measurements are not consistent within the errors. The difference between the two PAs used to apply the TW method is between $2^{\circ}$ and $7^{\circ}$ and it explains the different results for $\Omega_{\mathrm{bar}}$. Analysing the PA radial profiles in these cases we observed the photometric decomposition was not able to effectively describe the PA of the disc. As reference result, we adopted the value of $\Omega_{\text {bar }}$ obtained using the PA and $i$ defined from the radial profiles, as in previous works (Paper I; Cuomo et al. 2019). At the end of this analysis, seven more galaxies were discarded (Table 1) because the large errors on $\langle V\rangle(\Delta\langle V\rangle /\langle V\rangle>0.5$ translate into $\left.\Delta \Omega_{\mathrm{bar}} / \Omega_{\mathrm{bar}} \sim 1\right)$ or because the presence of residual spectral features in the CALIFA datacube prevented the convergence of $\langle X\rangle$ and/or $\langle V\rangle$.

The SDSS $r$ band images of the remaining 16 galaxies, which represent our sample of SAB galaxies successfully analysed with the TW method, are shown in Fig. 2. This means that $45 \%$ of the galaxies of the initial sample do not actually host a genuine bar component. The adopted values of PA and measurements of $\Omega_{\text {bar }}$ are listed in Table 2. Typical errors on $\Omega_{\text {bar }}$ are around $25 \%$. The TW integrals and best-fitting straight lines are plotted in Fig. 3.

\subsection{Corotation radius and bar rotation rate}

Although the TW method does not need any modelling to derive $\Omega_{\text {bar }}$, we need to make some assumptions to derive the circular velocity $V_{\text {circ }}$ and consequently $R_{\text {cr }}$ and $\mathcal{R}$.

We obtained $V_{\text {circ }}$ from the maps of stellar LOS velocity and velocity dispersion in the disc region provided by Falcón-Barroso et al. (2017) using the asymmetric drift equation (Binney \& Tremaine 1987) and following the prescriptions of Debattista et al. (2002) and Aguerri et al. (2003). In particular, we assumed an exponential radial profile with the same scale length for the radial, azimuthal, and vertical components of the velocity dispersion $\sigma_{R}, \sigma_{\phi}, \sigma_{z}$, the epicyclic approximation 

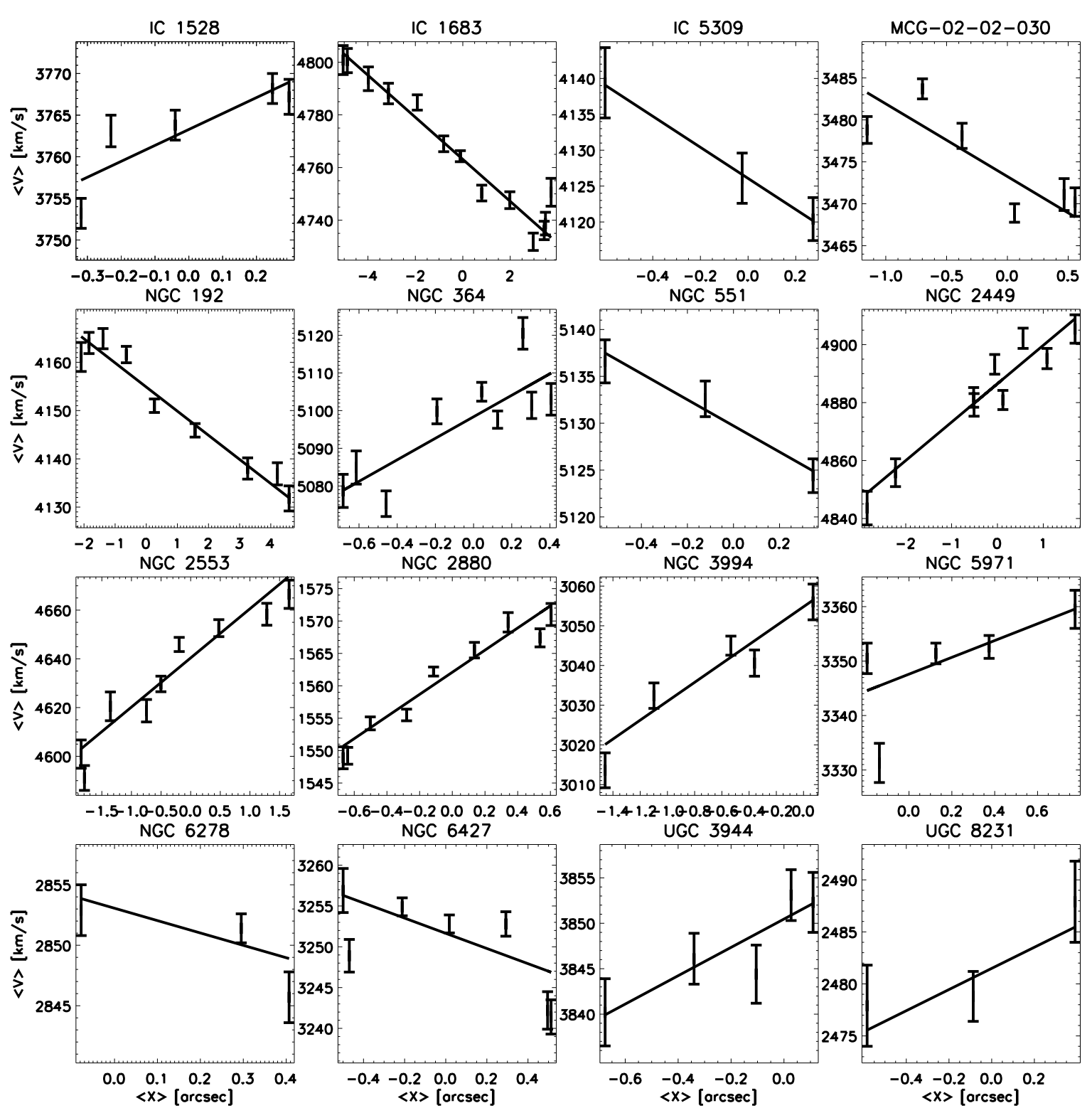

Fig. 3. Bar pattern speed of the $16 \mathrm{SAB}$ galaxies shown in Fig. 2. For each galaxy, the kinematic integrals $\langle V\rangle$ are plotted as a function of the photometric integrals $\langle X\rangle$ and the best-fitting straight line, which has a slope equal to $\Omega_{\mathrm{bar}} \sin i$, is overplotted.

and a constant $V_{\text {circ }}$ resulting in $\sigma_{\phi} / \sigma_{R}=1 / \sqrt{2}$, and a value for $\sigma_{z} / \sigma_{R}$ depending on the morphological type (Gerssen \& Shapiro Griffin 2012; Paper I).

We checked the reliability of our circular velocities by performing a comparison with the $V_{\text {circ }}$ values predicted by the Tully-Fisher relation of Reyes et al. (2011) and with those obtained by Leung et al. (2018) with different dynamical models from ours. Our galaxies are consistent within the $3 \sigma$ scatter of the relation between the circular velocity and absolute SDSS $r$ band magnitude calculated by Reyes et al. (2011) for a sample of $\sim 200$ nearby SDSS galaxies (Fig. 4). Eight galaxies in our sample are in common with the subsample of $\sim 50$ CALIFA galaxies studied by Leung et al. (2018). Our values of $V_{\text {circ }}$ are in agreement within the errors with their values.

We calculated $R_{\mathrm{cr}}=V_{\text {circ }} / \Omega_{\text {bar }}$ from the asymmetric-drift circular velocity and TW bar pattern speed and we derived the ratio of the corotation to bar radius $\mathcal{R}=R_{\mathrm{cr}} / R_{\mathrm{bar}}$. The values of $V_{\text {circ }}$, $R_{\mathrm{cr}}$, and $\mathcal{R}$ are reported in Table 2 .

\section{Results}

\subsection{Ultrafast bars}

Two galaxies with a TW-measured $\Omega_{\text {bar }}(13 \%)$ host an ultrafast bar having $\mathcal{R}<1$ at $95 \%$ confidence level (Table 2). This $\mathcal{R}$ regime corresponds to bars extending beyond $R_{\mathrm{cr}}$, which are expected to rapidly dissolve.

In Paper I, we explored possible explanations for measuring $\mathcal{R}<1$ with the TW method, which include obtaining the incorrect estimate of $R_{\text {bar }}$ and/or $R_{\mathrm{cr}}$, the application to objects which do not meet all the TW requirements, or the presence of multiple pattern speeds associated with the main bar, the spiral arms, and a nuclear bar. In order to address these issues, we obtained $R_{\text {bar }}$ with three different and independent methods. In some cases these estimates are quite different from each other, but this reflects the adopted error on $R_{\mathrm{bar}}$, which we defined as the largest difference between the mean value and the three measurements. On the other hand, $R_{\mathrm{cr}}$ depends on both $V_{\text {circ }}$ and $\Omega_{\mathrm{bar}}$. The circular velocity was obtained using the asymmetric drift 


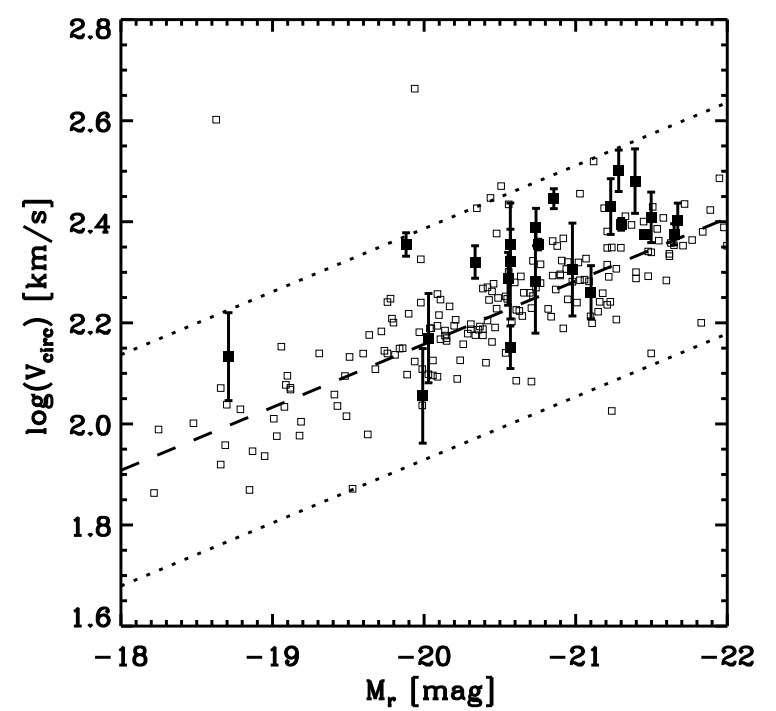

Fig. 4. Circular velocity as a function of absolute $r$ band magnitude of the SAB galaxies successfully analysed with the TW method (filled squares) and the galaxy sample of Reyes et al. (2011) (open squares). The dashed line is the best-fitting relation of Reyes et al. (2011) and the dotted lines bracket the region of $3 \sigma$ deviation in $\log \left(V_{\text {circ }}\right)$.

correction and the resulting values are consistent with the predictions of the Tully-Fisher relation (Reyes et al. 2011) and previous measurements based on different dynamical models (Leung et al. 2018). In Cuomo et al. (2019) we show that $\mathcal{R}<1$ could be the result of an incorrect estimate of the disc PA when the PA radial profile does not present a constant trend in the disc region. This is the reason why we checked the constancy of the profiles and finally used the PA from the photometric analysis to recover $\Omega_{\mathrm{bar}}$. A slope change with radius of the straight-line fitting $\langle X\rangle$ and $\langle V\rangle$ is interpreted as being due to components rotating with a different pattern speed with respect to the main bar (Corsini et al. 2003; Maciejewski 2006; Meidt et al. 2009). This change is observed in IC 1683, NGC 2553, and NGC 6427 (Fig. 3), although not all of them host an ultrafast bar (Table 2).

We ran a Kolmogorov-Smirnov (KS) test with the IDL procedure KSTWO to verify whether there are statistical differences between the distributions of morphological type, redshift, and absolute $r$ band magnitude of the initial sample of 29 bona fide SAB galaxies, the sample of $16 \mathrm{SAB}$ galaxies successfully analysed with the TW method, and the sample of 14 SAB galaxies without an ultrafast bar (Fig. 1). Since we found no significant difference at a very high confidence level $(>95 \%)$ in the properties of the three samples, we decided to not consider further the ultrafast bars.

\subsection{Bar properties in weakly and strongly barred galaxies}

Our goal is to compare the bar properties of a sample of SB and SAB galaxies with a TW-measured $\Omega_{\text {bar }}$ and of their host galaxies. To perform an effective comparison, the different bar properties have to be derived using similar methodologies within the sample. To this end, we added ESO-139-G0009 (Aguerri et al. 2003) to our sample of SAB galaxies without an ultrafast bar, and as a comparison sample of SB galaxies we collected 17 (Debattista \& Williams 2004; Paper I; Cuomo et al. 2019) and 15 galaxies (Merrifield \& Kuijken 1995; Gerssen et al. 1999, 2003; Debattista et al. 2002; Aguerri et al. 2003; Corsini et al. 2003, 2007; Treuthardt et al. 2007) with $\Omega_{\text {bar }}$ measured with the TW method from the stellar kinematics obtained with integral-field and long-slit spectroscopy, respectively. Only 3 out of the 32 SB galaxies host an ultrafast bar (9\%). In Fig. 5 we show the distributions of morphological type of the SAB and SB galaxies including or excluding the ultrafast bars. The remarkably large number of SB0 galaxies is an effect of the selection bias due to the application of the TW method to early-type disc galaxies with a low dust and gas content (see Corsini 2011, for a review).

We investigated the distributions of absolute $r$ band magnitude and bar properties of SAB and SB galaxies without an ultrafast bar, respectively. For each parameter we performed a KS test to look for statistically significant differences between the two samples. We found that the bars in SAB galaxies are similar to those of SB for all the explored parameters; in particular, the two samples have similar bar strengths. This is due to the fact that visually classified SAB galaxies are contaminated by strong bars while comparing the strength of the bar. The mean strength value of the SAB galaxies is $\left\langle S_{\text {bar }}\right\rangle=0.42 \pm 0.18$. On the other hand, visually classified SB galaxies may host weak bars in terms of the strength (Fig. 6). This suggests that a visual separation between weak and strong bars does not correspond to classifying the galaxies according to the bar strength.

Since a quantitative distinction between strong and weak bars has not been defined (Athanassoula 2003; Athanassoula et al. 2013; Vera et al. 2016; Kruk et al. 2018), we decided to split our full sample of 46 galaxies using a quantitative criterion based on the strength, if available. The chosen limiting value is $S_{\text {bar }}=0.4$, which means including $50 \%$ of visually classified $\mathrm{SAB}$ galaxies in the new quantitative defined $\mathrm{SAB}$ sample and having enough objects in SB and SAB samples to perform some significant statistics. Speaking about strong and weak bars, from now on we refer to quantitative $\mathrm{SB}$ and $\mathrm{SAB}$ galaxies because their definition is based on the strength.

We investigated the distributions of bar properties and absolute $r$ band magnitudes of the quantitatively defined 13 SAB and 27 SB galaxies classified through the bar strength and without an ultrafast bar (Figs. 7 and 8). For each parameter, we performed a KS test to look for statistically significant differences between the two samples. We confirmed that the bars of SAB galaxies are weaker that those of SB galaxies, although their hosts have the same luminosity distribution. In addition, we found at a very high confidence level $(>99 \%)$ that weak bars are shorter and have smaller $R_{\mathrm{cr}}$ with respect to their strong counterparts. On the other hand, SAB and SB galaxies display similar distributions of $\Omega_{\mathrm{bar}}$ and the bar rotation rate. We repeated the analysis using the results obtained with the PA from the photometric decomposition and we obtained similar results. The relations between bar and galaxy properties are investigated in the following section.

\subsection{Bulge and disc properties in weakly and strongly barred galaxies}

We analysed the relations between the bar parameters and the bulge and disc properties of the SAB and SB galaxies. We recovered the bulge-to-total luminosity ratio $B / T$, bulge Sérsic parameter $n$, effective radius $R_{\mathrm{e}}$ of the bulge, and scale length $h$ of the disc from Méndez-Abreu et al. (2017) for the CALIFA galaxies and from the quoted papers for the other galaxies.

We performed a KS test on the bulge properties and found that SB and SAB galaxies present the same distributions for $n$ and $R_{\mathrm{e}}$ of the bulges at a high significance level, but have different distributions for $B / T$. Moreover, two $\mathrm{SAB}$ galaxies turned out to be bulgeless, while the result of the KS test on bulge 


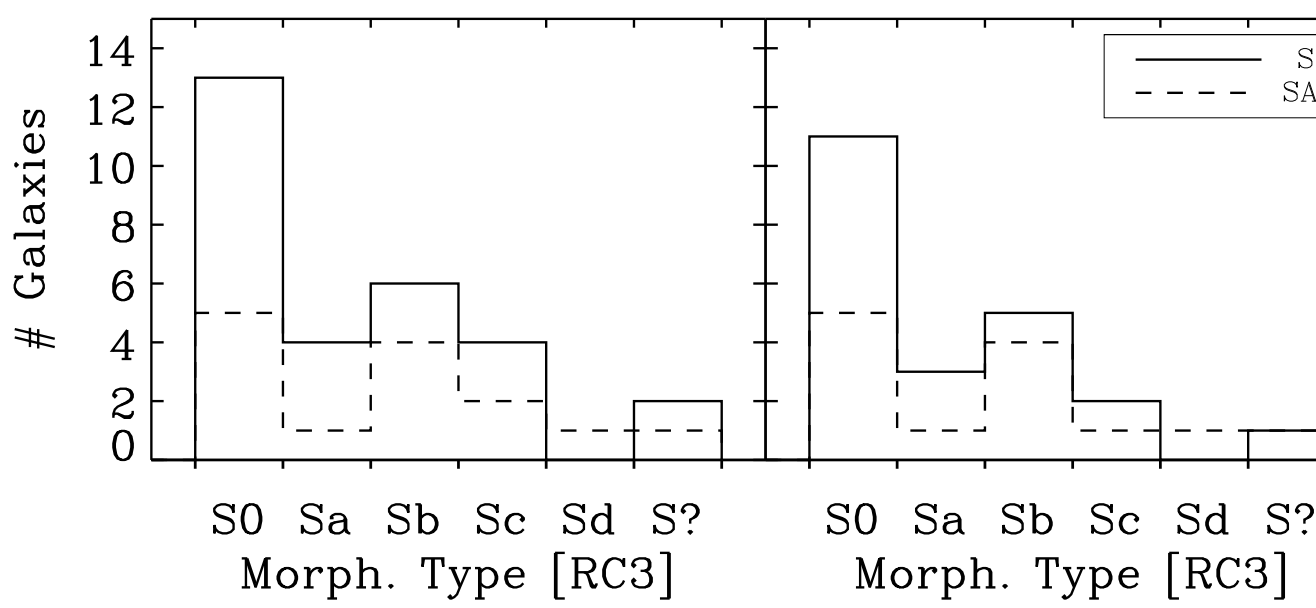

Fig. 5. Distributions of the morphological type of the SB (solid line) and SAB (dashed line) galaxies including (left panel) and excluding (right panel) the ultrafast bars.
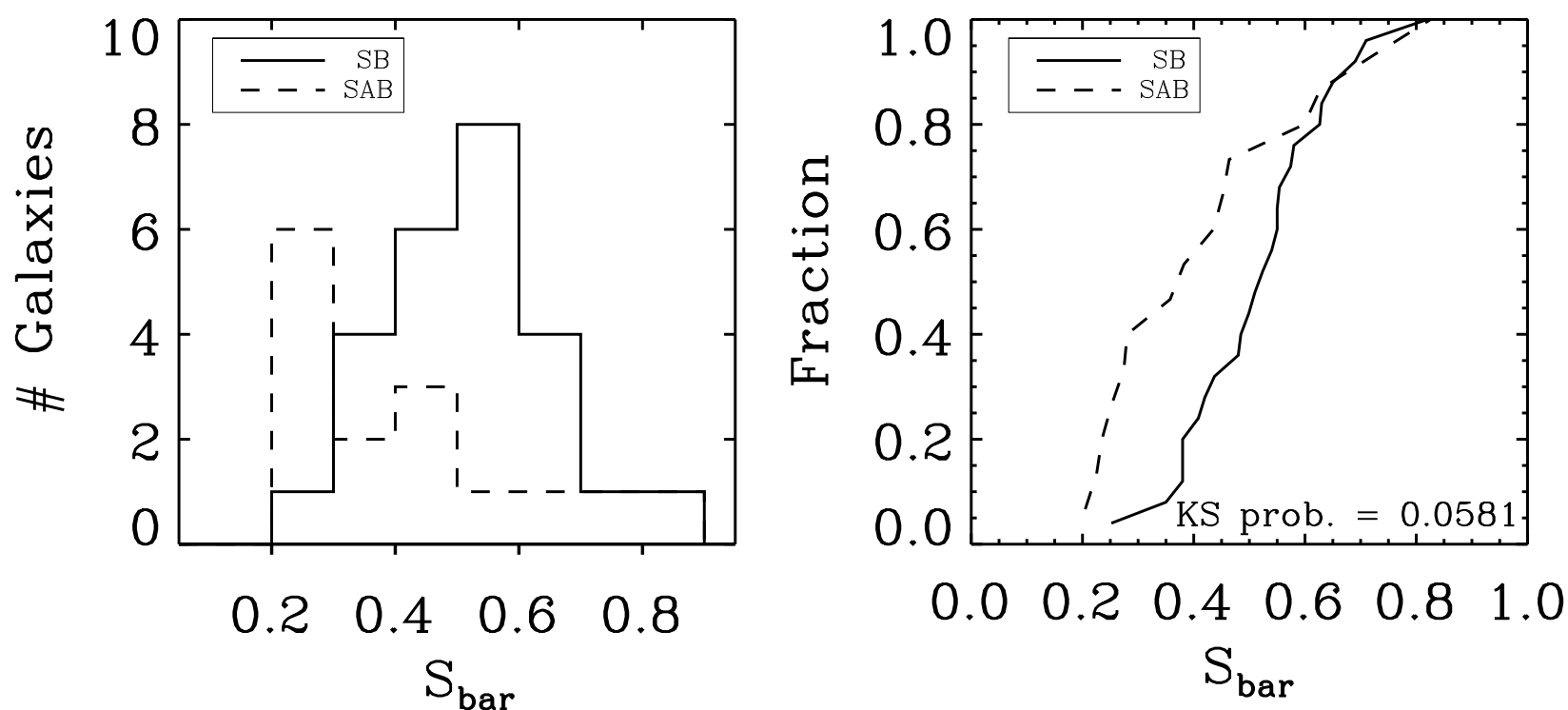

Fig. 6. Distribution of the bar strength of the SB (solid line) and SAB (dashed line) galaxies excluding the ultrafast bars (left panel) and cumulative distributions of SB (solid line) and SAB (dashed line) galaxies without any ultrafast bars as a function of bar strength (right panel).

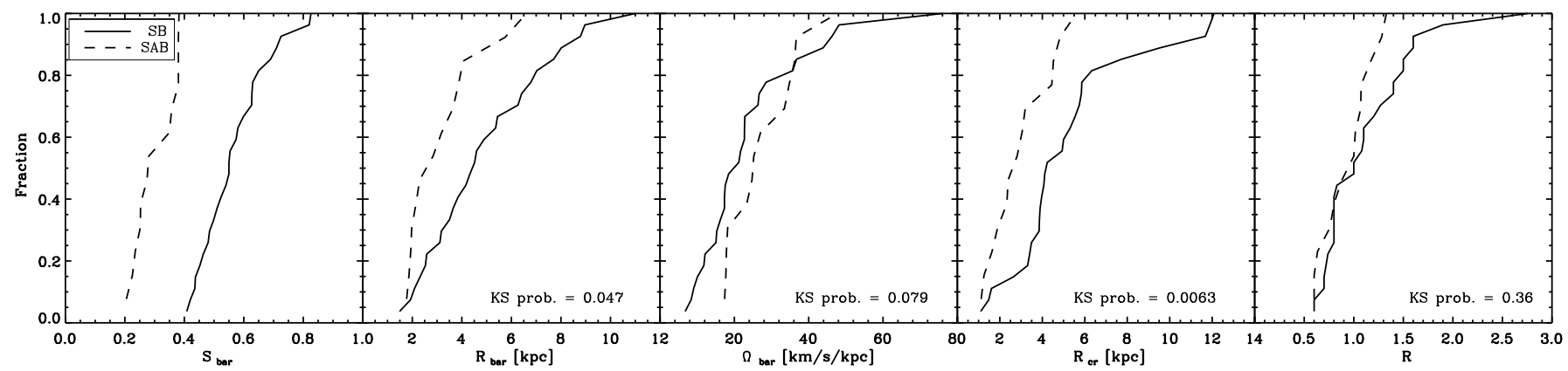

Fig. 7. Cumulative distributions of SB (solid line) and SAB (dashed line) galaxies without an ultrafast bar as a function of bar radius, bar strength, bar pattern speed, corotation radius, and bar rotation rate (from left to right). The significance level of the KS test is given in each panel.

properties remains the same even when discarding these two objects. This analysis suggests that bulges of SAB and SB galaxies present similar properties, but make a different contribution to the total light of their host galaxies.

The discs of SAB and SB galaxies are also similar to each other (Fig. 9). To investigate the disc regions hosting weak and strong bars, we measured the ratios between $R_{\mathrm{cr}}$ and $h$ and between $R_{\mathrm{bar}}$ and $h$ for the SAB and SB galaxies (Fig. 10). Most of the bars and corotation radii of the two galaxy samples are confined within or are close to their disc scale length since $R_{\mathrm{cr}} / h$ and $R_{\mathrm{bar}} / h$ typically range between 1 and 1.5. In particular, for SAB galaxies both $R_{\mathrm{bar}} / h$ and $R_{\mathrm{cr}} / h$ are lower than 1.5 (except 


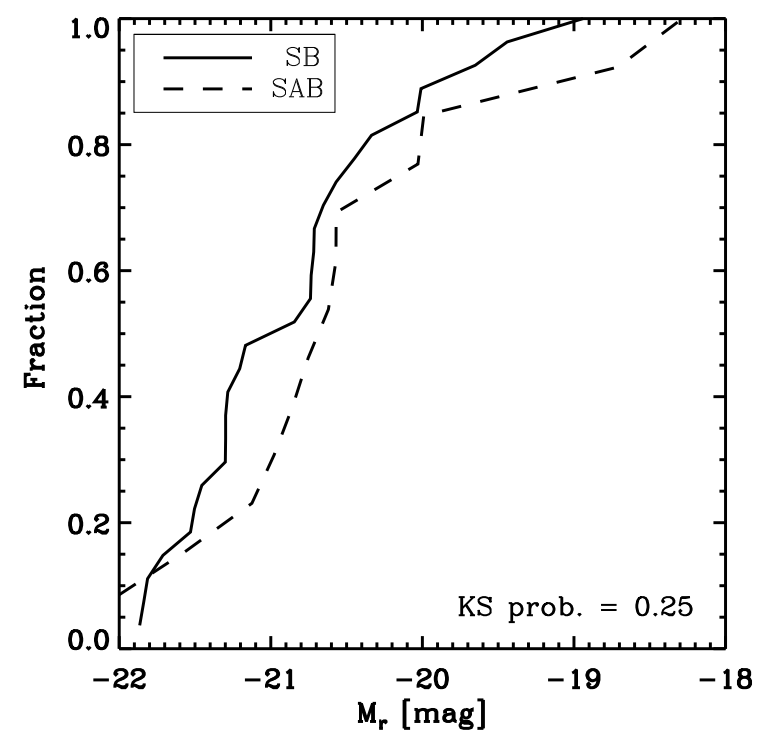

Fig. 8. Cumulative distributions of SB (solid line) and SAB (dashed line) galaxies without an ultrafast bar as a function of absolute $r$ band magnitude. The significance level of the KS test is given.

for one outlier whose $S_{\text {bar }}$ lies near the limiting value adopted to split the sample), while $30 \%$ of SB galaxies are characterised by $R_{\mathrm{cr}} / h$ and $R_{\mathrm{bar}} / h$ higher than 1.5 . The ratio of $R_{\mathrm{cr}} / h$ and $R_{\mathrm{bar}} / h$ corresponds to $\mathcal{R}$, which ranges between 1 and 1.4 corresponding to the fast bar regime.

\section{Discussion and conclusions}

In this work, we obtained the bar properties of a sample of 29 bona fide SAB galaxies by analysing the $r$ band images available from the SDSS survey and stellar kinematic maps obtained from the CALIFA survey (Table 2). The galaxies were selected to have an intermediate inclination, a bar elongated between the minor and major axes of the disc, and to be morphologically and kinematically undisturbed. The sample galaxies have morphological types ranging from S0 to Scd, with redshifts between 0.005 and 0.30 and absolute $r$ band total magnitudes from -18.5 to $-23.0 \mathrm{mag}$.

We derived the bar radius $R_{\mathrm{bar}}$ in the deprojected images of the galaxies by measuring the bar-interbar intensity ratio obtained from the Fourier analysis of the surface brightness distribution, the location of the maximum in the $\epsilon$ radial profile and the behaviour of the PA radial profile of the ellipses fitting the galaxy isophotes. At the same time, we measured the bar strength $S_{\text {bar }}$ from the Fourier analysis. Despite the stringent criteria we adopted for the selection, we discarded six galaxies because they did not host a clear bar component. The $m=2$ Fourier component did not show the amplitude peak with a constant phase angle typical of barred galaxies, while the large odd components revealed the presence of non-axisymmetric structures other than a bar.

We applied the TW method to obtain the bar pattern speed $\Omega_{\text {bar }}$ from the CALIFA datacubes. This study represents the third effort to apply the TW method to a large sample of galaxies based on integral-field spectroscopy, and the first to include SAB galaxies. To this end, we measured the luminosity-weighted mean position and LOS velocity of the stars across the bar in several pseudoslits parallel to the disc major axis. We rejected seven more galaxies because of the poor correlation, or the large errors, or the non-convergence of the TW integrals. This means that 13 galaxies in the sample $(45 \%)$, which were morphologically classified as weakly barred from a visual inspection, either do not actually host a bar component or have a central elongated structure that is not in rigid rotation. For the remaining $16 \mathrm{SAB}$ galaxies, we derived the corotation radius $R_{\mathrm{cr}}$ from the circular velocity obtained by applying the asymmetric drift correction to the stellar kinematics and the bar rotation rate $\mathcal{R}$ as the ratio between $R_{\mathrm{cr}}$ and $R_{\mathrm{bar}}$. All the measured SAB bars are consistent with being fast within the errors $(1<\mathcal{R}<1.4)$, except for two, which are ultrafast $(\mathcal{R}<1)$ at the $95 \%$ confidence level (Table 2 ) and were not considered further. Although several ultrafast bars have been found with the TW method using integral-field spectroscopic data (Paper I; Guo et al. 2019), their dynamics has not yet been fully explained and requires a deeper analysis both from an observational and theoretical point of view.

We built a comparison sample of SB galaxies with TWbased $\Omega_{\text {bar }}$ from the literature (Fig. 5). We split the entire sample of 46 barred galaxies (visually classified SB + SAB) analysed with the TW method according to the strength of the bar (if available) and excluding the ultrafast galaxies. The value $S_{\text {bar }}=0.4$ is adopted to provide a quantitative definition of SAB and SB galaxies, and the final sample includes 13 quantitative SAB and 27 quantitative SB galaxies. The SAB galaxies host weaker and shorter bars with smaller corotations than bars of SB galaxies. In the end, both SAB and SB galaxies have similar large pattern speeds and bar rotation rates, and therefore host fast bars (Fig. 7). After checking that the two samples do have similar absolute total magnitudes, we excluded that this result is due to a bias in the distribution of their luminosities (Fig. 8). Since SAB galaxies, similarly to SB galaxies, host fast bars, we can exclude that their formation was tidally triggered by a past interaction with a companion. The numerical simulations by Martinez-Valpuesta et al. (2017) and Łokas (2018) show that tidally induced bars suffer a steady weakening across their evolution but their rotation rate is always in the slow regime. Our SAB sample includes many early-type disc galaxies (Fig. 5), which were found to host fast bars in earlier studies (e.g. Rautiainen et al. 2008; Font et al. 2017). However, we did not find a significant correlation between $\mathcal{R}$ and morphological type because of the small number statistics.

Since one of the most promising and often advocated causes of bar weakening is the presence of a central mass concentration, we investigated the relation between the presence of weak or strong bars and the bulge properties of the host galaxy. We did not find any significant difference in the Seŕsic index $n$ and effective radius $R_{\mathrm{e}}$ of the bulges of SAB and SB galaxies. Instead we find a lower values of $B / T$ in SAB galaxies. Moreover, we found two bulgeless SAB galaxies. A similar result was found by Abraham \& Merrifield (2000), who showed that SAB galaxies are less concentrated than their SB counterparts. Therefore, we conclude that the presence of a prominent bulge does not necessarily imply the bar weakening. Moreover, we clearly found that $\Omega_{\text {bar }}$ of weak and strong bars is similar, as previously suggested by measurements with other methods (Font et al. 2017). This allowed us to discard the dissolution scenarios, which always predict an increase in $\Omega_{\text {bar }}$ while the bar is losing strength and dissolving, regardless of different causes of dissolution, such as the presence of central mass concentration, shape of DM halo, or gas accretion (Athanassoula 2003; Bournaud et al. 2005; Athanassoula et al. 2005). Laurikainen et al. (2013) suggested that bulges in the early-type SB galaxies are built by bars, while those in the SAB galaxies are possibly the end result a several accretion events that occurred before the bar formation, prescribing different values for the $n$ index. In our sample this 


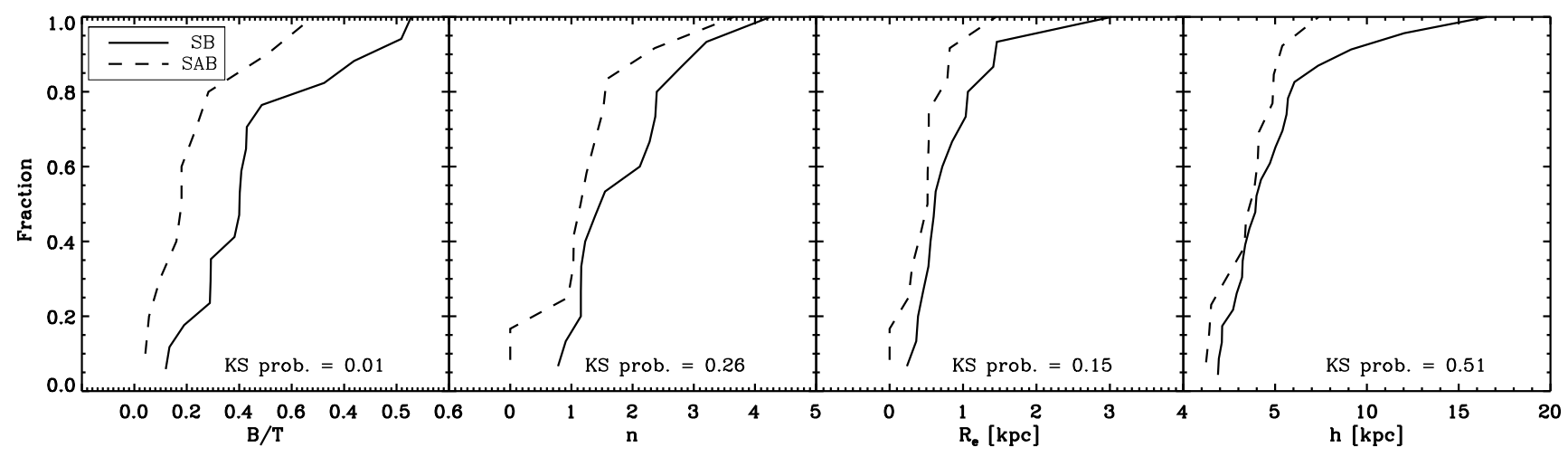

Fig. 9. Cumulative distributions of SB (solid line) and SAB (dashed line) galaxies without an ultrafast bar as a function of bulge-to-total luminosity, bulge Sérsic index, and bulge effective radius; cumulative distributions of SB and SAB galaxies without an ultrafast bar as a function of disc scale length (from left to right). The significance level of the KS test is given in each panel.

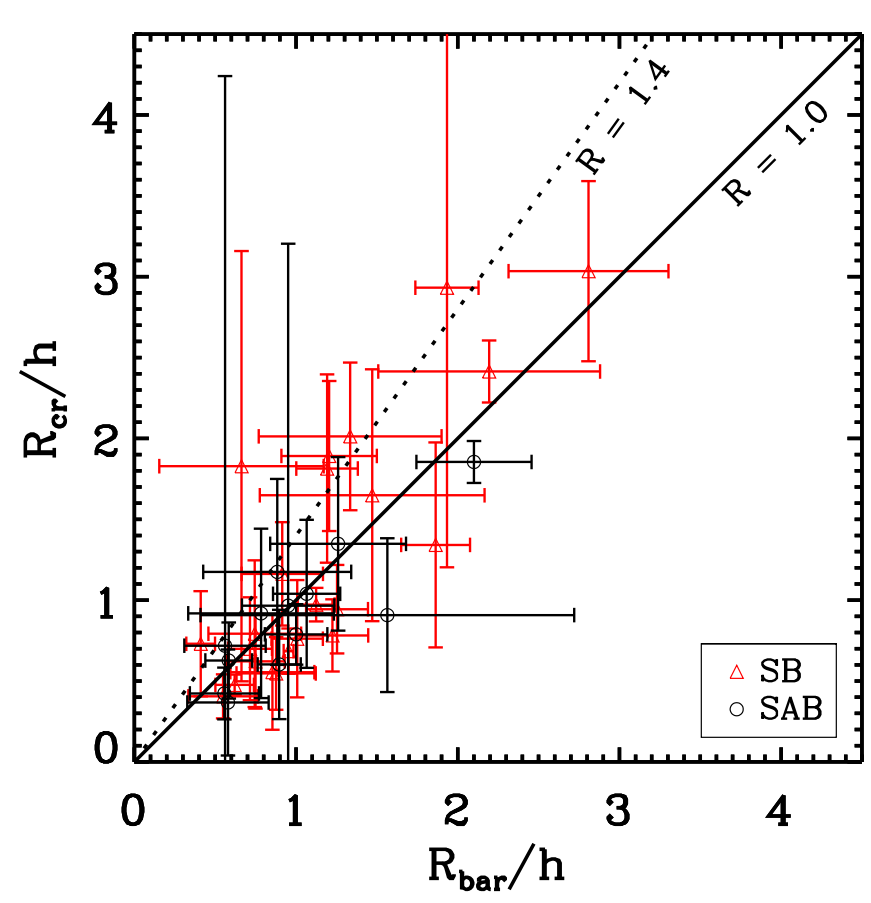

Fig. 10. Ratio of the corotation radius to disc scale length as a function of the ratio between the bar radius and disc scale length for SB (red triangles) and SAB (black circles) galaxies without an ultrafast bar. The solid and dashed lines indicate $\mathcal{R}=1.0$ and 1.4 , respectively.

formation mechanism is not supported because we observe the same distribution of $n$ in SB and SAB galaxies. We can not further investigate the bulge type in SB and SAB galaxies because the Sérsic index $n$ does not provide a clear separation between classical and pseudobulges and a variety of spectroscopic and photometric diagnostics including the bulge intrinsic shape is needed (Costantin et al. 2017, 2018; Méndez-Abreu et al. 2018).

We explored the relation between the presence of a weak or strong bar and the disc scale length of the host galaxy. We found that weak bars are all hosted in the inner parts of discs because most SAB galaxies have both $R_{\mathrm{bar}} / h$ and $R_{\mathrm{cr}} / h$ lower than 1.0 and in all SAB galaxies these ratios are lower than 1.5, except for one outlier. We observed a wider spread of $R_{\text {bar }} / h$ and $R_{\mathrm{cr}} / h$ for SB galaxies, with a clear tail to values larger than 1.5 (Fig. 10).

A generalised picture for bar formation and evolution can be summarised as follows. A bar in the early stage of evolution extends to the corotation $(\mathcal{R} \sim 1)$ and presents a high value of
$\Omega_{\mathrm{bar}}$. Then, both $R_{\mathrm{bar}}$ and $R_{\mathrm{cr}}$ increase as a consequence of the angular momentum exchange between the bar and other galactic components, while $\Omega_{\text {bar }}$ decreases. At some point during the evolution, the corotation reaches the disc region where the star density is too low to further feed the bar. From this moment, $R_{\mathrm{cr}}$ increases more than $R_{\mathrm{bar}}$ and the rotation rate is expected to enter the slow regime $(\mathcal{R}>1.4)$ (Debattista et al. 2006; Athanassoula et al. 2013).

In this scenario, $\mathrm{SAB}$ galaxies with low $R_{\mathrm{bar}} / h$ and $R_{\mathrm{cr}} / h$ could be young bars, while SB galaxies with high $R_{\text {bar }} / h$ and $R_{\text {cr }} / h$ could be old bars. However, SB and SAB galaxies present similar values of $\Omega_{\text {bar }}$ and none of the bars analysed in this work or in previous TW-based works are unambiguously located in the slow regime. Moreover, it is very unlikely to catch a bar in its early phase of evolution because the bar formation phase is very short. All this evidence suggests that SAB galaxies are dynamically evolved systems that did not exchange as much angular momentum as the SB galaxies and their hosting bars have not grown, while the paucity of slow bars remains unexplained. To confirm this scenario, further observations, dynamical modelling, and numerical simulations focused onto SAB galaxies are required because it is known that the exchange of angular momentum between the bar and other components depends on several parameters including the DM central concentration (e.g. Debattista \& Sellwood 2000), initial gas fraction, and halo triaxiality (e.g. Athanassoula et al. 2013), disc thickness (e.g. Klypin et al. 2009), and stellar mass distribution, and/or weak interactions not always clearly visible in the velocity fields (e.g. Salak et al. 2019).

Acknowledgements. We thank the anonymous referee for the constructive suggestions. We are grateful to M. Bureau, P. T. de Zeeuw, J. Falcón-Barroso, F. Pinna, L. Costantin, I. Pagotto, and M. Rubino for their valuable comments. V.C. acknowledges support from the Fondazione Ing. Aldo Gini and thanks the Instituto de Astrofísica de Canarias and the Universidad de la Laguna for hospitality during the preparation of this paper. V.C., E.M.C., and A.P. are supported by Padua University through grants DOR1715817/17, DOR1885254/18, DOR1935272/19, and BIRD164402/16. J.A.L.A. and J.M.A. are supported by the Spanish MINECO grants AYA2017-83204-P and AYA2013-43188-P. V.P.D. is supported by STFC Consolidated grant ST/R000786/1.

\section{References}

Abazajian, K. N., Adelman-McCarthy, J. K., Agüeros, M. A., et al. 2009, ApJS, 182,543

Abolfathi, B., Aguado, D. S., Aguilar, G., et al. 2018, ApJS, 235, 42

Abraham, R. G., \& Merrifield, M. R. 2000, AJ, 120, 2835 
V. Cuomo et al.: Bar pattern speeds in CALIFA galaxies. II.

Abraham, R. G., Merrifield, M. R., Ellis, R. S., Tanvir, N. R., \& Brinchmann, J. 1999, MNRAS, 308, 569

Aguerri, J. A. L., Muñoz-Tuñón, C., Varela, A. M., \& Prieto, M. 2000, A\&A, 361,841

Aguerri, J. A. L., Debattista, V. P., \& Corsini, E. M. 2003, MNRAS, 338, 465

Aguerri, J. A. L., Méndez-Abreu, J., \& Corsini, E. M. 2009, A\&A, 495, 491

Aguerri, J. A. L., Méndez-Abreu, J., Falcón-Barroso, J., et al. 2015, A\&A, 576, A102

Athanassoula, E. 1992, MNRAS, 259, 345

Athanassoula, E. 2003, MNRAS, 341, 1179

Athanassoula, E., \& Misiriotis, A. 2002, MNRAS, 330, 35

Athanassoula, E., Lambert, J. C., \& Dehnen, W. 2005, MNRAS, 363, 496

Athanassoula, E., Machado, R. E. G., \& Rodionov, S. A. 2013, MNRAS, 429 1949

Barazza, F. D., Jogee, S., \& Marinova, I. 2008, ApJ, 675, 1194

Barway, S., Wadadekar, Y., \& Kembhavi, A. K. 2011, MNRAS, 410, L18

Binney, J., \& Tremaine, S. 1987, Galactic Dynamics (Princeton: Princeton University Press)

Bournaud, F., \& Combes, F. 2002, A\&A, 392, 83

Bournaud, F., Combes, F., \& Semelin, B. 2005, MNRAS, 364, L18

Bundy, K., Bershady, M. A., Law, D. R., et al. 2015, ApJ, 798, 7

Buta, R. J., \& Zhang, X. 2009, ApJS, 182, 559

Buta, R. J., Corwin, H. G., \& Odewahn, S. C. 2007, The de Vaucouleurs Atlas of Galaxies (Cambridge: Cambridge University Press)

Buta, R. J., Sheth, K., Athanassoula, E., et al. 2015, ApJS, 217, 32

Cappellari, M., \& Emsellem, E. 2004, PASP, 116, 138

Combes, F. 2011, Mem. Soc. Astron. It. Suppl., 18, 53

Contopoulos, G. 1981, A\&A, 102, 265

Contopoulos, G., \& Papayannopoulos, T. 1980, A\&A, 92, 33

Corsini, E. M. 2011, Mem. Soc. Astron. Ital. Suppl., 18, 23

Corsini, E. M., Debattista, V. P., \& Aguerri, J. A. L. 2003, ApJ, 599, L29

Corsini, E. M., Aguerri, J. A. L., Debattista, V. P., et al. 2007, ApJ, 659, L12

Costantin, L., Méndez-Abreu, J., Corsini, E. M., et al. 2017, A\&A, 601, A84

Costantin, L., Corsini, E. M., Méndez-Abreu, J., et al. 2018, MNRAS, 481, 3623

Cuomo, V., Corsini, E. M., Aguerri, J. A. L., et al. 2019, MNRAS, 488, 4972

de Lorenzo-Cáceres, A., Méndez-Abreu, J., Thorne, B., \& Costantin, L. 2019, MNRAS, 484, 665

de Vaucouleurs, G. 1959, Handbuch der Physik, 53, 275

de Vaucouleurs, G., de Vaucouleurs, A., Corwin, Jr., H. G., et al. 1991, Third Reference Catalogue of Bright Galaxies. Volume I: Explanations and references. Volume II: Data for galaxies between $0^{\mathrm{h}}$ and $12^{\mathrm{h}}$. Volume III: Data for galaxies between $12^{\mathrm{h}}$ and $24^{\mathrm{h}}$

Debattista, V. P. 2003, MNRAS, 342, 1194

Debattista, V. P., \& Sellwood, J. A. 1998, ApJ, 493, L5

Debattista, V. P., \& Sellwood, J. A. 2000, ApJ, 543, 704

Debattista, V. P., \& Williams, T. B. 2004, ApJ, 605, 714

Debattista, V. P., Corsini, E. M., \& Aguerri, J. A. L. 2002, MNRAS, 332, 65

Debattista, V. P., Mayer, L., Carollo, C. M., et al. 2006, ApJ, 645, 209

Erwin, P. 2018, MNRAS, 474, 5372

Falcón-Barroso, J., Lyubenova, M., van de Ven, G., et al. 2017, A\&A, 597, A48

Font, J., Beckman, J. E., Martínez-Valpuesta, I., et al. 2017, ApJ, 835, 279

Garcia-Gómez, C., Athanassoula, E., Barberà, C., \& Bosma, A. 2017, A\&A, 601, A132

Gerhard, O. E. 1993, MNRAS, 265, 213

Gerssen, J., \& Debattista, V. P. 2007, MNRAS, 378, 189

Gerssen, J., \& Shapiro Griffin, K. 2012, MNRAS, 423, 2726

Gerssen, J., Kuijken, K., \& Merrifield, M. R. 1999, MNRAS, 306, 926

Gerssen, J., Kuijken, K., \& Merrifield, M. R. 2003, MNRAS, 345, 261

Guo, R., Mao, S., Athanassoula, E., et al. 2019, MNRAS, 482, 1733

Hinshaw, G., Larson, D., Komatsu, E., et al. 2013, ApJS, 208, 19

Hirota, A., Kuno, N., Sato, N., et al. 2009, PASJ, 61, 441

Hohl, F. 1971, ApJ, 168, 343

Hubble, E. P. 1936, Realm of the Nebulae (New Haven: Yale University Press)

Husemann, B., Jahnke, K., Sánchez, S. F., et al. 2013, A\&A, 549, A87
Jedrzejewski, R. I. 1987, MNRAS, 226, 747

Klypin, A., Valenzuela, O., Colín, P., \& Quinn, T. 2009, MNRAS, 398, 1027

Kormendy, J. 1979, ApJ, 227, 714

Kruk, S. J., Lintott, C. J., Bamford, S. P., et al. 2018, MNRAS, 473, 4731

Laurikainen, E., \& Salo, H. 2002, MNRAS, 337, 1118

Laurikainen, E., Salo, H., Athanassoula, E., et al. 2013, MNRAS, 430, 3489

Lee, Y. H., Ann, H. B., \& Park, M.-G. 2019, ApJ, 872, 97

Leung, G. Y. C., Leaman, R., van de Ven, G., et al. 2018, MNRAS, 477, 254

Li, Z.-Y., Ho, L. C., \& Barth, A. J. 2017, ApJ, 845, 87

Lin, Y., Cervantes Sodi, B., Li, C., Wang, L., \& Wang, E. 2014, ApJ, 796, 98

Łokas, E. L. 2018, ApJ, 857, 6

Maciejewski, W. 2006, MNRAS, 371, 451

Manos, T., \& Athanassoula, E. 2011, MNRAS, 415, 629

Marinova, I., \& Jogee, S. 2007, ApJ, 659, 1176

Martinet, L., \& Udry, S. 1990, A\&A, 235, 69

Martinez-Valpuesta, I., Aguerri, J., \& González-García, C. 2016, Galaxies, 4, 7 Martinez-Valpuesta, I., Aguerri, J. A. L., González-García, A. C., Dalla Vecchia, C., \& Stringer, M. 2017, MNRAS, 464, 1502

Masters, K. L., Nichol, R. C., Hoyle, B., et al. 2011, MNRAS, 411, 2026

Meidt, S. E., Rand, R. J., \& Merrifield, M. R. 2009, ApJ, 702, 277

Méndez-Abreu, J., Aguerri, J. A. L., Corsini, E. M., \& Simonneau, E. 2008, A\&A, 478, 353

Méndez-Abreu, J., Sánchez-Janssen, R., \& Aguerri, J. A. L. 2010, ApJ, 711, L61

Méndez-Abreu, J., Sánchez-Janssen, R., Aguerri, J. A. L., Corsini, E. M., \& Zarattini, S. 2012, ApJ, 761, L6

Méndez-Abreu, J., Debattista, V. P., Corsini, E. M., \& Aguerri, J. A. L. 2014, A\&A, 572, A25

Méndez-Abreu, J., Ruiz-Lara, T., Sánchez-Menguiano, L., et al. 2017, A\&A, 598, A32

Méndez-Abreu, J., Aguerri, J. A. L., Falcón-Barroso, J., et al. 2018, MNRAS, 474, 1307

Merrifield, M. R., \& Kuijken, K. 1995, MNRAS, 274, 933

Nair, P. B., \& Abraham, R. G. 2010, ApJ, 714, L260

Noguchi, M. 1987, MNRAS, 228, 635

Ohta, K., Hamabe, M., \& Wakamatsu, K.-I. 1990, ApJ, 357, 71

Patsis, P. A., \& Katsanikas, M. 2014, MNRAS, 445, 3525

Press, W. H., Teukolsky, S. A., Vetterling, W. T., \& Flannery, B. P. 1992, Numerical Recipes in FORTRAN. The Art of Scientific Computing (Cambridge: Cambridge University Press)

Raha, N., Sellwood, J. A., James, R. A., \& Kahn, F. D. 1991, Nature, 352, 411

Rautiainen, P., Salo, H., \& Laurikainen, E. 2008, MNRAS, 388, 1803

Reyes, R., Mandelbaum, R., Gunn, J. E., Pizagno, J., \& Lackner, C. N. 2011 , MNRAS, 417, 2347

Roth, M. M., Kelz, A., Fechner, T., et al. 2005, PASP, 117, 620

Salak, D., Noma, Y., Sorai, K., et al. 2019, PASJ, 17

Sánchez, S. F., Kennicutt, R. C., Gil de Paz, A., et al. 2012, A\&A, 538, A8

Sánchez, S. F., García-Benito, R., Zibetti, S., et al. 2016, A\&A, 594, A36

Sánchez-Janssen, R., Méndez-Abreu, J., \& Aguerri, J. A. L. 2010, MNRAS, 406, L65

Sarzi, M., Falcón-Barroso, J., Davies, R. L., et al. 2006, MNRAS, 366, 1151

Sellwood, J. A. 1981, A\&A, 99, 362

Toomre, A. 1981, in Structure and Evolution of Normal Galaxies, eds. S. M. Fall, \& D. Lynden-Bell (Cambridge: Cambridge University Press), 111

Tremaine, S., \& Weinberg, M. D. 1984, ApJ, 282, L5

Treuthardt, P., Buta, R., Salo, H., \& Laurikainen, E. 2007, AJ, 134, 1195

Valdes, F., Gupta, R., Rose, J. A., Singh, H. P., \& Bell, D. J. 2004, ApJS, 152, 251

van den Bergh, S. 1976, ApJ, 206, 883

van der Marel, R. P., \& Franx, M. 1993, ApJ, 407, 525

Vasiliev, E., \& Athanassoula, E. 2015, MNRAS, 450, 2842

Vera, M., Alonso, S., \& Coldwell, G. 2016, A\&A, 595, A63

Walcher, C. J., Wisotzki, L., Bekeraité, S., et al. 2014, A\&A, 569, A1

Wozniak, H., \& Pierce, M. J. 1991, A\&AS, 88, 325 